\title{
FIXED POINTS OF HOLOMORPHIC MAPPINGS FOR DOMAINS IN BANACH SPACES
}

\author{
LAWRENCE A. HARRIS
}

Received 29 November 2001

We discuss the Earle-Hamilton fixed-point theorem and show how it can be applied when restrictions are known on the numerical range of a holomorphic function. In particular, we extend the Earle-Hamilton theorem to holomorphic functions with numerical range having real part strictly less than 1 . We also extend the Lumer-Phillips theorem estimating resolvents to dissipative holomorphic functions.

\section{Introduction}

Our purpose is to provide an introduction to holomorphic fixed-point theorems and to describe some recent joint work with Simeon Reich and David Shoikhet. The main results are a fixed-point theorem and a Lumer-Phillips theorem, which is a mapping theorem for resolvents. These theorems apply to holomorphic functions whose numerical range is a set of complex numbers that lies to the left of a certain vertical line.

We begin by recalling some basic facts about holomorphic functions with domain and range in a Banach space. We give two equivalent definitions of holomorphy (weak and strong) and discuss power series and Taylor's theorem. The holomorphic functional calculus is included as an example, although this is not needed later.

Next we give a brief overview of fixed-point theorems for holomorphic functions beginning with the Earle-Hamilton fixed-point theorem. This theorem may be viewed as a holomorphic version of Banach's contraction mapping theorem since a holomorphic function that maps a bounded domain strictly inside itself is a contraction with respect to a natural holomorphically invariant metric on the domain. (As usual, a contraction is a Lipschitzian mapping with Lipschitz constant less than 1.) Our formulation is slightly more general than the 
original since we require only the given function to be bounded rather than that its domain of definition.

We give a self-contained proof of the Earle-Hamilton theorem and deduce a closely related fixed-point theorem of Khatskevich, Reich, and Shoikhet. The latter theorem obtains a unique fixed point for a holomorphic function of a convex domain into itself provided that the function has a uniformly continuous extension to the closure of the domain and there are no sequences of approximate fixed points on its boundary.

Since the main results refer to the numerical range of holomorphic functions, we introduce this subject by beginning with the classical definition of the numerical range for operators on a Hilbert space. We then discuss Lumer's extension of the numerical range and its properties for linear operators on a Banach space. This leads to the author's extension to holomorphic functions on the open unit balls of Banach spaces. Finally, we give the general definition for holomorphic functions on convex domains containing the origin.

Our first main result is an extension of the Earle-Hamilton theorem. This asserts that (under mild restrictions) if the numerical range of a holomorphic function lies strictly to the left of the vertical line $x=1$, then the function has a unique fixed point in its domain. A consequence is that a uniformly continuous holomorphic function $h$ on a domain is dissipative (i.e., its numerical range lies on or to the left of the imaginary axis) if and only if the resolvent $(I-t h)^{-1}$ is defined on the domain and maps it into itself. In the case where $h$ is a linear operator on a Banach space, this reduces to a theorem of Lumer and Phillips.

\section{Holomorphic functions}

Holomorphic functions on abstract spaces were considered by M. Fréchet around 1910 and subsequently studied by many authors. (See $[3,4,14]$.) We give two definitions of holomorphy. Let $X$ and $Y$ be complex Banach spaces and let $\mathscr{D}$ be an open subset of $X$.

Definition 2.1 (strong definition). A function $h: \mathscr{D} \rightarrow Y$ is holomorphic if for each $x \in \mathscr{D}$ there exists a continuous complex-linear mapping $D h(x): X \rightarrow Y$ such that

$$
\lim _{y \rightarrow 0} \frac{\|h(x+y)-h(x)-D h(x) y\|}{\|y\|}=0 .
$$

There is an alternative weaker definition which reduces matters to the case of a complex-valued function of a complex variable. (We use $\lambda$ to denote a complex variable.)

Definition 2.2 (weak definition). A function $h: \mathscr{D} \rightarrow Y$ is holomorphic if it is locally bounded and if the mapping

$$
\lambda \longrightarrow \ell(h(x+\lambda y))
$$

is holomorphic at $\lambda=0$ for each $x \in \mathscr{D}, y \in X$ and linear functional $\ell \in Y^{*}$. 
Clearly the strong definition implies the weak definition. It was shown by Dunford in 1938, using the uniform boundedness principle, that both definitions are equivalent. (See [14, Theorems 3.10.1 and 3.17.1].) In many cases, $\mathscr{D}$ will be the open ball of radius $r$ and center $x$, that is,

$$
B_{r}(x)=\{z \in X:\|z-x\|<r\} .
$$

If $S$ is a set of complex numbers, we define

$$
|S|=\sup \{|\lambda|: \lambda \in S\} .
$$

Example 2.3. Define a mapping $P: X \rightarrow Y$ to be a homogeneous polynomial of degree $n$ if

$$
P(x)=F(\underbrace{x, \ldots, x}_{n})
$$

where $F: X \times \cdots \times X \rightarrow Y$ is a continuous (complex) multilinear map of degree $n$. Then $P$ is holomorphic on $X$ and

$$
D P(x) y=\sum_{k=0}^{n-1} F(\underbrace{x, \ldots, x}_{k}, y, \underbrace{x, \ldots, x}_{n-k-1}), \quad x, y \in X .
$$

If $\left\{P_{n}\right\}_{0}^{\infty}$ is a sequence of homogeneous polynomials where $P_{n}$ is of degree $n$ and if there exist positive constants $M$ and $r$ such that $\left\|P_{n}\right\| r^{n} \leq M$ for $n=0,1, \ldots$, then it follows easily from the Weierstrass $M$-test and the weak definition that

$$
h(x)=\sum_{n=0}^{\infty} P_{n}(x)
$$

converges to a holomorphic function in $B_{r}(0)$.

The $n$th derivative $D^{n} h(x)$ of $h$ at $x$ can be represented as a multilinear map of degree $n$. We denote the associated homogeneous polynomial of degree $n$ by $\hat{D}^{n} h(x)$. It can be shown that

$$
P_{n}=\frac{1}{n !} \hat{D}^{n} h(0), \quad n=0,1, \ldots
$$

The following theorem shows that every holomorphic function can be written as the sum of an infinite series of homogeneous polynomials in some neighborhood of each of the points of its domain.

Theorem 2.4 (Taylor's theorem [14, Theorem 3.17.1]). If $h$ is holomorphic and bounded in $B_{r}(x)$, then

$$
h(x+y)=\sum_{n=0}^{\infty} \frac{1}{n !} \hat{D}^{n} h(x)(y)
$$

for all $y$ in $B_{r}(0)$. 
Example 2.5. The holomorphic functional calculus associates classical holomorphic functions with holomorphic functions on domains in spaces of operators. Specifically, let $W$ be a complex Banach space and let $X=\mathscr{L}(W)$ be the Banach space of all bounded linear operators on $W$. Suppose $f(\lambda)$ is a complex-valued function holomorphic in the disk $|\lambda|<r$. Then

$$
f(\lambda)=\sum_{n=0}^{\infty} a_{n} \lambda^{n}, \quad \text { for }|\lambda|<r,
$$

and hence

$$
f(A)=\sum_{n=0}^{\infty} a_{n} A^{n}
$$

is defined for $|\sigma(A)|<r$, where

$$
|\sigma(A)|=\lim _{n \rightarrow \infty}\left\|A^{n}\right\|^{1 / n}
$$

is the spectral radius. It is not difficult to show that the set

$$
\mathscr{D}=\{A \in X:|\sigma(A)|<r\}
$$

is open and it follows from Example 2.3 that $f: \mathscr{D} \rightarrow X$ is holomorphic.

More generally, let $\Omega$ be an open subset of the complex plane and let

$$
\mathscr{D}=\{A \in X: \sigma(A) \subseteq \Omega\} .
$$

Define

$$
f(A)=\frac{1}{2 \pi i} \int_{\mathscr{C}} f(\lambda)(\lambda I-A)^{-1} d \lambda,
$$

where $\mathscr{C}$ consists of a finite number of positively oriented rectifiable Jordan curves in $\Omega$ whose interiors are a disjoint cover $\sigma(A)$. It can be shown (see [14, Theorem 5.2.4] or [5, page 568]) that this defines $f(A)$ independently of the curve $\mathscr{C}$ and the domain $\Omega$ and that $f: \mathscr{D} \rightarrow X$ is holomorphic.

Example 2.6. To justify hypotheses in later theorems, we exhibit a function that is holomorphic everywhere in $X=c_{0}$, bounded on $B_{r}(0)$ for every $r$ with $0<r<1$ but unbounded on $B_{1}(0)$. Define

$$
h(x)=\sum_{k=0}^{\infty} x_{k}^{k}, \quad \text { for } x=\left(x_{k}\right)_{0}^{\infty} .
$$

Then $h$ is defined and holomorphic everywhere in $X$ by the weak definition. Also, since $\left|x_{k}^{k}\right| \leq\|x\|^{k}$ for any $x \in X$, it follows that

$$
\|h(x)\| \leq \frac{1}{1-\|x\|}, \quad x \in B_{1}(0) .
$$


Now for each positive integer $n$ define an $x \in B_{1}(0)$ by

$$
\begin{aligned}
& x_{k}=\frac{1}{\sqrt[n]{2}}, \quad 0 \leq k \leq n, \\
& x_{k}=0, \quad k>n .
\end{aligned}
$$

Then

$$
h(x) \geq \sum_{k=0}^{n} x_{k}^{n} \geq \sum_{k=0}^{n} \frac{1}{2} .
$$

Hence $h$ is unbounded in $B_{1}(0)$.

\section{Holomorphic fixed-point theorems}

A set $S$ is said to lie strictly inside a subset $\mathscr{D}$ of a Banach space if there is some $\epsilon>0$ such that $B_{\epsilon}(x) \subseteq \mathscr{D}$ whenever $x \in S$. The following theorem may be viewed as a holomorphic version of the Banach's contraction mapping theorem.

Theorem 3.1 (Earle-Hamilton [6]). Let D be a nonempty domain in a complex Banach space $X$ and let $h: \mathscr{D} \rightarrow \mathscr{D}$ be a bounded holomorphic function. If $h(\mathscr{D})$ lies strictly inside D, then $h$ has a unique fixed point in $\mathscr{D}$.

Proof. We construct a metric $\rho$, called the CRF-pseudometric, in which $h$ is a contraction. Define

$$
\alpha(x, v)=\sup \{|D g(x) v|: g: \mathscr{D} \longrightarrow \Delta \text { holomorphic }\}
$$

for $x \in \mathscr{D}$ and $v \in X$, and set

$$
L(\gamma)=\int_{0}^{1} \alpha\left(\gamma(t), \gamma^{\prime}(t)\right) d t
$$

for $\gamma$ in the set $\Gamma$ of all curves in $\mathscr{D}$ with piecewise continuous derivative. Clearly $\alpha$ specifies a seminorm at each point of $\mathscr{D}$. We view $L(\gamma)$ as the length of the curve $\gamma$ measured with respect to $\alpha$. Define

$$
\rho(x, y)=\inf \{L(\gamma): \gamma \in \Gamma, \gamma(0)=x, \gamma(1)=y\}
$$

for $x, y \in \mathscr{D}$. It is easy to verify that $\rho$ is a pseudometric on $\mathscr{D}$.

Let $x \in \mathscr{D}$ and $v \in X$. By the chain rule,

$$
D(g \circ h)(x) v=D g(h(x)) D h(x) v,
$$

for any holomorphic function $g: \mathscr{D} \rightarrow \Delta$. Hence,

$$
\alpha(h(x), D h(x) v) \leq \alpha(x, v) .
$$


By integrating this and applying the chain rule, we obtain $L(h \circ \gamma) \leq L(\gamma)$ for all $\gamma \in \Gamma$ and thus the Schwarz-Pick inequality

$$
\rho(h(x), h(y)) \leq \rho(x, y)
$$

holds for all $x, y \in \mathscr{D}$.

Now by hypothesis there exists an $\epsilon>0$ such that $B_{\epsilon}(h(x)) \subseteq \mathscr{D}$ whenever $x \in \mathscr{D}$. We may assume that $\mathscr{D}$ is bounded by replacing $\mathscr{D}$ by the subset

$$
\cup\left\{B_{\epsilon}(h(x)): x \in \mathscr{D}\right\}
$$

Fix $t$ with $0<t<\epsilon / \delta$, where $\delta$ denotes the diameter of $h(\mathscr{D})$. Given $x \in \mathscr{D}$, define

$$
\hat{h}(y)=h(y)+t[h(y)-h(x)]
$$

and note that $\hat{h}: \mathscr{D} \rightarrow \mathscr{D}$ is holomorphic. Given $x \in \mathscr{D}$ and $v \in X$, it follows from

$$
D \hat{h}(x) v=(1+t) D h(x) v
$$

and (3.5), with $h$ replaced by $\hat{h}$, that

$$
\alpha(h(x), D h(x) v) \leq \frac{1}{1+t} \alpha(x, v) .
$$

Integrating this as before, we obtain

$$
\rho(h(x), h(y)) \leq \frac{1}{1+t} \rho(x, y)
$$

for all $x, y \in \mathscr{D}$.

Now pick a point $x_{0} \in \mathscr{D}$ and let $\left\{x_{n}\right\}$ be the sequence of iterates given by $x_{n}=h^{n}\left(x_{0}\right)$. Then $\left\{x_{n}\right\}$ is a $\rho$-Cauchy sequence by the proof of the contraction mapping theorem. Since $X$ is complete in the norm metric, it suffices to show that there exists a constant $m>0$ such that

$$
\rho(x, y) \geq m\|x-y\|,
$$

for all $x, y \in \mathscr{D}$. Since $\mathscr{D}$ is bounded, we may take $m=1 / d$, where $d$ is the diameter of $\mathscr{D}$. Given $x \in \mathscr{D}$ and $v \in X$, define

$$
g(y)=m \ell(y-x)
$$

where $\ell \in X^{*}$ with $\|\ell\|=1$. Then $g: \mathscr{D} \rightarrow \Delta$ is holomorphic and $D g(x) v=m \ell(v)$. Hence $\alpha(x, v) \geq m\|v\|$ by the Hahn-Banach theorem. Integrating as before, we obtain (3.12).

The Earle-Hamilton theorem still applies in cases where the holomorphic function does not necessarily map its domain strictly inside itself. In fact, the 
following interesting fixed-point theorem is a consequence of two applications of the Earle-Hamilton theorem.

Theorem 3.2 (Khatskevich-Reich-Shoikhet $[15,19]$ ). Let $\mathscr{D}$ be a nonempty bounded convex domain in a Banach space and let $h: \mathscr{D} \rightarrow \mathscr{D}$ be a holomorphic function having a uniformly continuous extension to $\overline{\mathscr{D}}$. If there exists an $\epsilon>0$ such that $\|h(x)-x\| \geq \epsilon$ whenever $x \in \partial \mathscr{D}$, then $h$ has a unique fixed point in $\mathscr{D}$.

For example, the hypothesis that $\|h(x)-x\| \geq \epsilon$ for all $x \in \partial \mathscr{D}$ is satisfied when $\mathscr{D}$ contains the origin and

$$
\sup _{x \in \partial D} \frac{\|h(x)\|}{\|x\|}<1 .
$$

Proof. Given $0<t<1$ and $x \in \mathscr{D}$, define

$$
f_{t}(y)=(1-t) x+t h(y)
$$

and let $\delta>0$ be such that $B_{\delta}(x) \subseteq \mathscr{D}$. Since $\mathscr{D}$ is convex, $f_{t}: \mathscr{D} \rightarrow \mathscr{D}$ is holomorphic. To show that $f_{t}(\mathscr{D})$ lies strictly inside $\mathscr{D}$, take $\epsilon=(1-t) \delta$. Let $y \in \mathscr{D}$ and let $w \in B_{\epsilon}\left(f_{t}(y)\right)$. Then

$$
z=\frac{w-t h(y)}{1-t}
$$

is in $\mathscr{D}$ since $z \in B_{\delta}(x)$, so

$$
w=(1-t) z+t h(y) \in \mathscr{D} .
$$

Hence $B_{\epsilon}\left(f_{t}(y)\right) \subseteq \mathscr{D}$ for all $y \in \mathscr{D}$.

By the Earle-Hamilton theorem, $f_{t}$ has a unique fixed point $g_{t}(x)$ in $\mathscr{D}$. Since the CRF-metric is continuous, the proof of the contraction mapping theorem shows that the iterates of $f_{t}$ at a chosen point $y_{0} \in \mathscr{D}$ are holomorphic and locally uniformly Cauchy in $x$. Hence the limit function $g_{t}: \mathscr{D} \rightarrow \mathscr{D}$ is holomorphic by [14, Theorem 3.18.1]. Now an $x \in \mathscr{D}$ is a fixed point for $g_{t}$ if and only if $x$ is a fixed point for $h$. Thus, by the Earle-Hamilton theorem, it suffices to show that $g_{t}(\mathscr{D})$ lies strictly inside $\mathscr{D}$ for some $t>0$.

Since $h$ has a uniformly continuous extension to $\overline{\mathscr{D}}$, there exist $\epsilon>0$ and $\delta>0$ such that $\|h(x)-x\| \geq \epsilon$ whenever $x \in \mathscr{D}$ and

$$
d(x, \partial \mathscr{D})=\inf \{\|x-y\|: y \in \partial \mathscr{D}\}<\delta .
$$

Since $\mathscr{D}$ is bounded, there is an $M$ with $\|x\| \leq M$ for all $x \in \mathscr{D}$. If $x \in \mathscr{D}$,

$$
h\left(g_{t}(x)\right)-g_{t}(x)=(1-t)\left[h\left(g_{t}(x)\right)-x\right],
$$

so

$$
\left\|h\left(g_{t}(x)\right)-g_{t}(x)\right\| \leq 2(1-t) M .
$$


Choose $t$ close enough to 1 so that $2(1-t) M<\epsilon$. If $d\left(g_{t}(x), \partial \mathscr{D}\right)<\delta$ for some $x \in \mathscr{D}$, then

$$
\epsilon \leq\left\|h\left(g_{t}(x)\right)-g_{t}(x)\right\|
$$

a contradiction. Thus, $B_{\delta}\left(g_{t}(x)\right) \subseteq \mathscr{D}$ for all $x \in \mathscr{D}$, as required.

Considerably stronger results have been obtained for the case where $\mathscr{D}$ is the open unit ball of a Hilbert space.

Theorem 3.3 (Goebel-Sekowski-Stachura [9, Theorem 11]). Let B be the open unit ball of a Hilbert space and let $h: B \rightarrow B$ be holomorphic. If there is a point $x_{0}$ in $B$ such that the sequence $\left\{h^{n}\left(x_{0}\right)\right\}$ of iterates lies strictly inside $B$, then $h$ has a fixed point in $B$.

Obtaining fixed points for mappings that are only nonexpansive rather than contractive is more difficult. A fundamental result due, independently, to Browder, Göhde, and Kirk in 1965 is that a nonexpansive self-mapping of a closed bounded convex set in a uniformly convex Banach space has a fixed point. In analogy, Goebel, Sekowski, and Stachura [9] showed that the CRF-metric $\rho$ in the open unit ball $B$ of a Hilbert space is uniformly convex and obtained a fixedpoint theorem for holomorphic self-mappings of $B$. All such mappings are $\rho$ nonexpansive by the Schwarz-Pick inequality (3.6).

Theorem 3.4 (Goebel-Reich [8, Theorem 25.4]). Let B be the open unit ball of a Hilbert space and let $h: B \rightarrow B$ be an arbitrary function satisfying

$$
\rho(h(x), h(y)) \leq \rho(x, y)
$$

for all $x, y \in B$. If h has a continuous extension to $\bar{B}$, then $h$ has a fixed point in $\bar{B}$.

Corollary 3.5 (Goebel-Sekowski-Stachura [9]). If $h: B \rightarrow B$ is a holomorphic function that has a continuous extension to $\bar{B}$, then $h$ has a fixed point in $\bar{B}$.

Extensions of Theorems 3.3 and 3.4 and of Corollary 3.5 to Cartesian products of the Hilbert ball $B$ can be found in papers of Kuczumow and others. It would be desirable to obtain extensions to more general spaces. See $[4,7,12]$ for basic material about function-theoretic metrics on domains in Banach spaces. See [16] for an extensive survey of fixed-point theorems for holomorphic mappings.

\section{The linear numerical range}

Let $A \in \mathscr{L}(H)$, where $H$ is a Hilbert space. The numerical range of $A$ is defined by

$$
W(A)=\{(A x, x):\|x\|=1, x \in H\} .
$$


Like the spectrum, the numerical range associates a set of complex numbers with $A$ that reflects the properties of $A$. However, unlike the spectral radius, the numerical radius is an equivalent norm on $\mathscr{L}(H)$. The following is a list of some basic properties. (See [10].)

Theorem 4.1. Let $A \in \mathscr{L}(H)$. Then

(1) $W(A)$ is convex,

(2) $A$ is hermitian (i.e., $A^{*}=A$ ) if and only if $W(A)$ is real,

(3) $\sigma(A) \subseteq \overline{W(A)}$,

(4) $|W(A)| \leq\|A\| \leq 2|W(A)|$.

The notion of the numerical range was successfully extended to operators on an arbitrary Banach space $X$ by Lumer [17]. To give an equivalent form of his definitions, define

$$
J(x)=\left\{\ell \in X^{*}:\|\ell\|=\ell(x)=1\right\}
$$

for $x \in X$ with $\|x\|=1$ and note that $J(x)$ is nonempty by the Hahn-Banach theorem. Let $Q(x)$ be a nonempty subset of $J(x)$ for each $x \in X$ with $\|x\|=1$.

Now let $A \in \mathscr{L}(X)$. Define numerical ranges of $A$ by

$$
\begin{aligned}
V(A) & =\{\ell(A x): \ell \in J(x),\|x\|=1\}, \\
W(A) & =\{\ell(A x): \ell \in Q(x),\|x\|=1\} .
\end{aligned}
$$

Clearly, $W(A) \subseteq V(A)$. If $Q(x)$ is taken to be $J(x)$ for all $x \in X$ with $\|x\|=1$, then $W(A)=V(A)$.

In the case where $X$ is a Hilbert space, the sets $Q(x)$ and $J(x)$ coincide and consist of the single functional $\ell(y)=(y, x)$ by the Riesz representation theorem. Thus $W(A)$ and $V(A)$ reduce to the previous definition of the numerical range.

Since there is in general no adjoint operation on $\mathscr{L}(X)$, an operator $A \in \mathscr{L}(X)$ is defined to be hermitian if $V(A)$ is real. The following theorem is a well-known extension of Theorem 4.1. (See Bonsall and Duncan [1,2] for this and many other properties of the numerical range.)

Theorem 4.2. Let $A \in \mathscr{L}(X)$. Then

(1) $V(A)$ is connected,

(2) $A$ is hermitian if and only if $\left\|e^{i t A}\right\|=1$ for all real $t$,

(3) $\overline{\mathrm{co}} \sigma(A) \subseteq \overline{V(A)}$,

(4) $|V(A)| \leq\|A\| \leq e|V(A)|$,

(5) $\sup \operatorname{Re} W(A)=\lim _{t \rightarrow 0^{+}}(\|I+t A\|-1) / t$.

In the above, the symbol $\overline{c o}$ denotes the closed convex hull. Following Lumer, we can deduce from part (5) of the above theorem that the closed convex hulls of $W(A)$ and $V(A)$ are equal no matter what choice of $Q$ is taken. Thus, in particular, $|W(A)|=|V(A)|$. 
The theorem we extend is a characterization of dissipative operators. This can be viewed as a one-sided formulation of part (2) of the previous theorem.

Theorem 4.3 (Lumer-Phillips [18]). Let $A \in \mathscr{L}(X)$. The following are equivalent:
(a) $\sup \operatorname{Re} W(A) \leq 0$,
(b) $\left\|e^{t A}\right\| \leq 1$ for all $t \geq 0$,
(c) $\left\|(I-t A)^{-1}\right\| \leq 1$ for all $t \geq 0$.

\section{The holomorphic numerical range}

We first consider the case where the domain is the open unit ball $B$ of $X$ and $h: B \rightarrow X$ is a holomorphic function that is uniformly continuous in $B$. Then $h$ has a uniformly continuous extension to $\bar{B}$. Moreover, $h$ is bounded in $B$ so

$$
\|h\|=\sup \{\|h(x)\|: x \in B\}
$$

is finite. In analogy with (4.3), define numerical ranges of $h$ by

$$
\begin{aligned}
V(h) & =\{\ell(h(x)): \ell \in J(x),\|x\|=1\}, \\
W(h) & =\{\ell(h(x)): \ell \in Q(x),\|x\|=1\} .
\end{aligned}
$$

Theorem 5.1 [11]. (a) $\sup \operatorname{Re} W(h)=\lim _{t \rightarrow 0^{+}}(\|I+t h\|-1) / t$.

(b) If $P_{n}$ is a homogeneous polynomial of degree $n>1$, then

$$
\left\|P_{n}\right\| \leq n^{n /(n-1)}\left|W\left(P_{n}\right)\right| .
$$

As was shown in [11], a consequence of part (a) of Theorem 5.1 is that the closed convex hulls of $W(h)$ and $V(h)$ are the same no matter what choice of $Q$ is taken. It was also shown in [11] that the constant in part (b) of the theorem is best possible.

Following [13], we now extend the numerical range to functions defined on more general domains. Let $\mathscr{D}$ be a convex domain in $X$ and suppose $\mathscr{D}$ contains the origin. For each $x \in \partial \mathscr{D}$, let

$$
J(x)=\left\{\ell \in X^{*}: \ell(x)=1, \operatorname{Re} \ell(y) \leq 1 \forall y \in \mathscr{D}\right\} .
$$

It follows from [5, Corollary 6, page 449] that $J(x)$ is nonempty. Let $Q(x)$ be a nonempty subset of $J(x)$ for each $x \in X$ with $x \in \partial \mathscr{D}$. If $h: \mathscr{D} \rightarrow X$ has a continuous extension to $\overline{\mathscr{D}}$, then we define

$$
\begin{aligned}
V(h) & =\{\ell(h(x)): \ell \in J(x), x \in \partial \mathscr{D}\}, \\
W(h) & =\{\ell(h(x)): \ell \in Q(x), x \in \partial \mathscr{D}\} .
\end{aligned}
$$

Otherwise, consider

$$
h_{s}(x)=h(s x), \quad 0<s<1 .
$$


The function $h_{s}$ always has a continuous extension to $\overline{\mathscr{D}}$ and hence we may define

$$
L(h)=\lim _{r \rightarrow 1^{-}} \sup \left\{\operatorname{Re} \ell\left(h_{s}(x)\right): \ell \in Q(x), x \in \partial \mathscr{D}, r \leq s<1\right\}
$$

since the indicated supremum is decreasing in $r$. If $h$ is uniformly continuous on $\mathscr{D}$, then $h$ has a uniformly continuous extension to $\overline{\mathscr{D}}$. Hence, $W(h)$ is defined and $L(h)=\sup \operatorname{Re} W(h)$.

Lemma 5.2 (cf. [11, Lemma 2]). If $h: \mathscr{D} \rightarrow X$ is holomorphic and bounded on each domain strictly inside $\mathscr{D}$, then $h_{s}$ is uniformly continuous on $\overline{\mathscr{D}}$ for each $s$ with $0<s<1$.

The following lemma is the key to our extension of the Lumer-Phillips theorem to holomorphic functions. Throughout the remainder of this section we assume that the domain $\mathscr{D}$ is bounded as well as convex.

Lemma 5.3 [13]. Let $g: \mathscr{D} \rightarrow X$ be holomorphic and bounded on each domain strictly inside $\mathscr{D}$. If $L(g)<0$, then the equation $g(x)=0$ has a unique solution $x \in \mathscr{D}$.

Proof. By Lemma 5.2, it suffices to prove the lemma for the case where $g$ is uniformly continuous. Let

$$
p(x)=\inf \{r>0: x \in r \mathscr{D}\}
$$

be the Minkowski functional for $\mathscr{D}$. It is not difficult to extend the proof of part (a) of Theorem 5.1 (given in [11]) to the case where $B$ is replaced by $\mathscr{D}$ and $p$ replaces the norm, that is,

$$
\|I+\operatorname{tg}\|=\sup \{p(x+\operatorname{tg}(x)): x \in \mathscr{D}\} .
$$

(In fact, we can avoid inverses in that proof by arguing as in [1, page 83].) By hypothesis there is an $\epsilon>0$ with sup $\operatorname{Re} W(g)<-\epsilon$. Hence there is a $t>0$ with

$$
p(x+\operatorname{tg}(x))<1-t \epsilon,
$$

for all $x \in \mathscr{D}$. Thus $(I+\operatorname{tg})(\mathscr{D})$ lies inside $(1-t \epsilon) \mathscr{D}$ and so strictly inside $\mathscr{D}$. By the Earle-Hamilton theorem, $I+\operatorname{tg}$ has a unique fixed point in $\mathscr{D}$ so $g(x)=0$ has a unique solution in $\mathscr{D}$.

Theorem 5.4 [13]. Let $h: \mathscr{D} \rightarrow X$ be holomorphic and bounded on each domain strictly inside $\mathscr{D}$. If $L(h)<1$, then $h$ has a unique fixed point in $\mathscr{D}$.

Proof. This theorem follows from the previous lemma with $g=h-I$ since $L(g)=$ $L(h)-1<0$.

The above theorem is an extension of the Earle-Hamilton fixed-point theorem since it is not difficult to show that $L(h)<1$ when $h$ maps $\mathscr{D}$ strictly inside D. 
Our main result is the following extension of the Lumer-Phillips theorem.

Theorem 5.5 [13]. Let $h: \mathscr{D} \rightarrow X$ be holomorphic and uniformly continuous. Then sup $\operatorname{Re} W(h) \leq 0$ if and only if $(I-t h)^{-1}$ is defined on $\mathscr{D}$ and is a holomorphic mapping of $\mathscr{D}$ into itself for each $t \geq 0$.

Proof. Suppose that sup $\operatorname{Re} W(h) \leq 0$. Since sup $\operatorname{Re} W(h)$ is positive homogeneous, it suffices to consider the case where $t=1$. Let $y \in \mathscr{D}$ and take $g=y+h$. Then

$$
\sup \operatorname{Re} W(g) \leq p(y)+\sup \operatorname{Re} W(h)<1
$$

by hypothesis. Hence by the previous theorem, the function $g$ has a unique fixed point $f(y)$ in $\mathscr{D}$. It can be shown as in the proof of Theorem 3.2 that $f$ is holomorphic in $\mathscr{D}$. Moreover, for each $y \in \mathscr{D}$, the only solution of $(I-h)(x)=y$ with $x \in \mathscr{D}$ is $x=f(y)$. Hence $(I-h)^{-1}=f$ exists on $\mathscr{D}$ and is a holomorphic mapping of $\mathscr{D}$ into itself.

Conversely, suppose that $(I-t h)^{-1}$ is defined on $\mathscr{D}$ and maps $\mathscr{D}$ into itself for all $t>0$. Since $h$ is uniformly continuous on $\mathscr{D}$ and $\mathscr{D}$ is bounded, there is an $M>0$ with $\|h(x)\| \leq M$ for all $x \in \mathscr{D}$. Also, given $\epsilon>0$ there exists a $\delta>0$ such that $\|h(x)-h(y)\|<\epsilon$ whenever $x, y \in \mathscr{D}$ and $\|x-y\|<\delta$. Clearly,

$$
(I-t h)^{-1}(x)-x=t h\left((I-t h)^{-1}(x)\right)
$$

for any $x \in \mathscr{D}$ and $t>0$. Let $0<t<\delta / M$. If $x \in \mathscr{D}$, then

$$
\left\|(I-t h)^{-1}(x)-x\right\|<\delta,
$$

so

$$
\left\|h\left((I-t h)^{-1}(x)\right)-h(x)\right\|<\epsilon .
$$

Now the Minkowski functional $p$ for $\mathscr{D}$ satisfies $p(x) \leq\|x\| / r$ for all $x \in X$, where $r>0$ is chosen so that $B_{r}(0) \subseteq \mathscr{D}$. Since $p$ is subadditive, it follows from (5.14) and the identity

$$
(I+t h)(x)=(I-t h)^{-1}(x)+t\left[h(x)-h\left((I-t h)^{-1}(x)\right)\right]
$$

that

$$
p((I+t h)(x)) \leq p\left((I-t h)^{-1}(x)\right)+t \frac{\epsilon}{r}
$$

for all $x \in \mathscr{D}$. Hence

$$
\|I+t h\| \leq 1+t \frac{\epsilon}{r}
$$

for $0<t<\delta / M$ and thus sup $\operatorname{Re} V(h) \leq \epsilon / r$. 
The above arguments obtain the forward implication of Theorem 5.5 as an easy consequence of Lemma 5.3. Conversely, Lemma 5.3 is an easy consequence of the forward implication of Theorem 5.5. To see this, by hypothesis and Lemma 5.2 we may suppose that $g: \mathscr{D} \rightarrow X$ has a uniformly continuous extension to $\overline{\mathscr{D}}$ and that

$$
\delta \equiv-\sup \operatorname{Re} W(g)>0
$$

Put $h=g+\delta I$ and note that sup Re $W(h) \leq 0$. Hence by Theorem 5.5, the equation $(I-t h)(x)=0$ has a unique solution $x$ in $\mathscr{D}$ for each $t \geq 0$. Since $g=$ $-\delta(I-t h)$ when $t=1 / \delta$, it follows that $g(x)=0$ has a unique solution $x$ in $\mathscr{D}$.

It is shown in [13, Corollary 9] that if $\mathscr{D}$ is the open unit ball of $X$ and if $L(h)$ is finite for the choice $Q=J$, then $h$ is bounded on each domain strictly inside $\mathscr{D}$. Thus, in the case mentioned, this hypothesis may be omitted from Lemma 5.3 and Theorem 5.4.

See [13] for further details and applications to Bloch's theorem and Cartan's uniqueness theorem.

In the case where the underlying space is finite dimensional, there is a version of Lemma 5.3 where the domain $\mathscr{D}$ does not need to be convex and the numerical range is computed as in a Hilbert space. (For purposes of comparison, we reformulate the theorem.)

Theorem 5.6 (Shih [20]). Suppose $X$ is finite dimensional and let $(\cdot, \cdot)$ be an inner product on $X$. Let $\mathscr{D}$ be a bounded domain in $X$ containing the origin and let $g: \mathscr{D} \rightarrow X$ be a holomorphic function with a continuous extension to $\overline{\mathscr{D}}$. If $\operatorname{Re}(g(x), x)<0$ for all $x \in \partial \mathscr{D}$, then $g(x)=0$ has a unique solution $x$ in $\mathscr{D}$.

\section{References}

[1] F. F. Bonsall and J. Duncan, Numerical Ranges of Operators on Normed Spaces and of Elements of Normed Algebras, London Mathematical Society Lecture Note Series, vol. 2, Cambridge University Press, Cambridge, 1971.

[2] - Numerical Ranges. II, London Mathematical Society Lecture Notes Series, no. 10, Cambridge University Press, Cambridge, 1973.

[3] S. Dineen, Complex Analysis in Locally Convex Spaces, North-Holland Mathematics Studies, vol. 57, North-Holland Publishing, Amsterdam, 1981.

[4] The Schwarz Lemma, Oxford Mathematical Monographs, Oxford University Press, New York, 1989.

[5] N. Dunford and J. T. Schwartz, Linear Operators. Part I, Wiley Classics Library, John Wiley \& Sons, New York, 1988.

[6] C. J. Earle and R. S. Hamilton, A fixed point theorem for holomorphic mappings, Global Analysis (Proc. Sympos. Pure Math., Vol. XVI, Berkeley, Calif., (1968)), American Mathematical Society, Rhode Island, 1970, pp. 61-65.

[7] C. J. Earle, L. A. Harris, J. H. Hubbard, and S. Mitra, Schwarz's lemma and the Kobayashi and Carathéodory metrics on complex Banach manifolds, Kleinian Groups and Hyperbolic 3-Manifolds, London Mathematical Society Lecture Note Series, Cambridge University Press, Cambridge, to appear. 
[8] K. Goebel and S. Reich, Uniform Convexity, Hyperbolic Geometry, and Nonexpansive Mappings, Monographs and Textbooks in Pure and Applied Mathematics, vol. 83, Marcel Dekker, New York, 1984.

[9] K. Goebel, T. Sekowski, and A. Stachura, Uniform convexity of the hyperbolic metric and fixed points of holomorphic mappings in the Hilbert ball, Nonlinear Anal. 4 (1980), no. 5, 1011-1021.

[10] K. E. Gustafson and D. K. M. Rao, Numerical Range, Universitext, Springer-Verlag, New York, 1997.

[11] L. A. Harris, The numerical range of holomorphic functions in Banach spaces, Amer. J. Math. 93 (1971), 1005-1019.

[12] Schwarz-Pick systems of pseudometrics for domains in normed linear spaces, Advances in Holomorphy (Proc. Sem. Univ. Fed. Rio de Janeiro, Rio de Janeiro, 1977) (J. A. Barroso, ed.), North-Holland Math. Stud., vol. 34, North-Holland Publishing, Amsterdam, 1979, pp. 345-406.

[13] L. A. Harris, S. Reich, and D. Shoikhet, Dissipative holomorphic functions, Bloch radii, and the Schwarz lemma, J. Anal. Math. 82 (2000), 221-232.

[14] E. Hille and R. S. Phillips, Functional Analysis and Semi-Groups, American Mathematical Society Colloquium Publications, vol. 31, American Mathematical Society, Rhode Island, 1957.

[15] V. Khatskevich, S. Reich, and D. Shoikhet, Fixed point theorems for holomorphic mappings and operator theory in indefinite metric spaces, Integral Equations Operator Theory 22 (1995), no. 3, 305-316.

[16] T. Kuczumow, S. Reich, and D. Shoikhet, Fixed points of holomorphic mappings: a metric approach, Handbook of Metric Fixed Point Theory (W. A. Kirk and B. Sims, eds.), Kluwer Academic Publishers, Dordrecht, 2001, pp. 437-515.

[17] G. Lumer, Semi-inner-product spaces, Trans. Amer. Math. Soc. 100 (1961), 29-43.

[18] G. Lumer and R. S. Phillips, Dissipative operators in a Banach space, Pacific J. Math. 11 (1961), 679-698.

[19] S. Reich and D. Shoikhet, Generation theory for semigroups of holomorphic mappings in Banach spaces, Abstr. Appl. Anal. 1 (1996), no. 1, 1-44.

[20] M. H. Shih, Bolzano's theorem in several complex variables, Proc. Amer. Math. Soc. 79 (1980), no. 1, 32-34.

Lawrence A. Harris: Department of Mathematics, University of Kentucky, Lexington, KY 40506, USA

E-mail address: larry@ms.uky.edu 


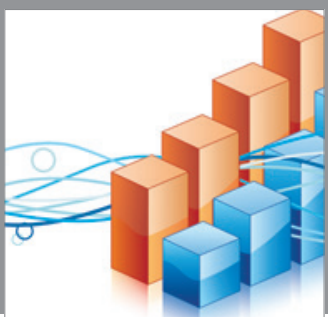

Advances in

Operations Research

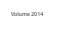

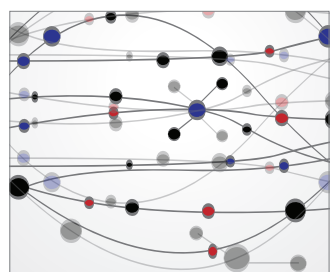

\section{The Scientific} World Journal
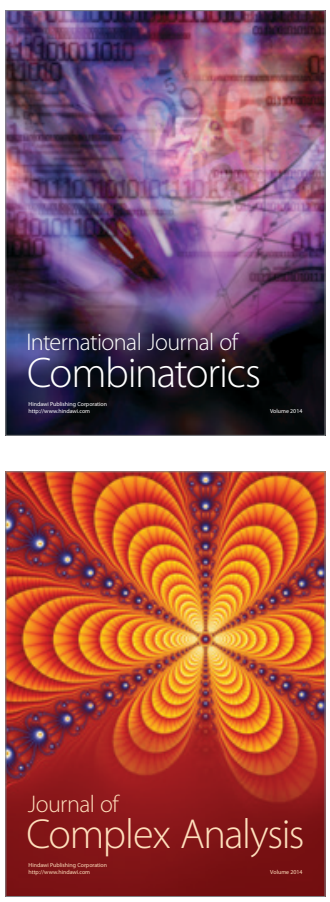

International Journal of

Mathematics and

Mathematical

Sciences
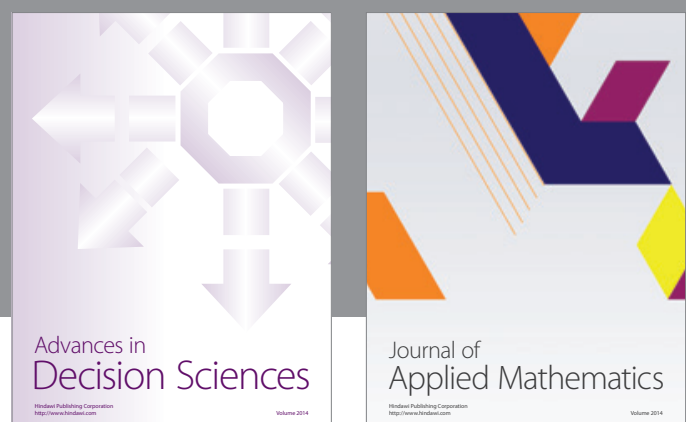

Journal of

Applied Mathematics
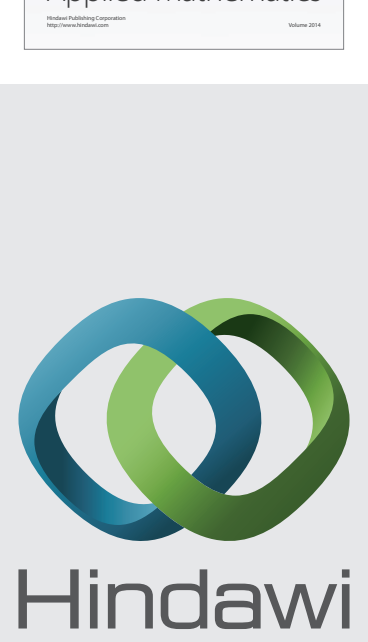

Submit your manuscripts at http://www.hindawi.com
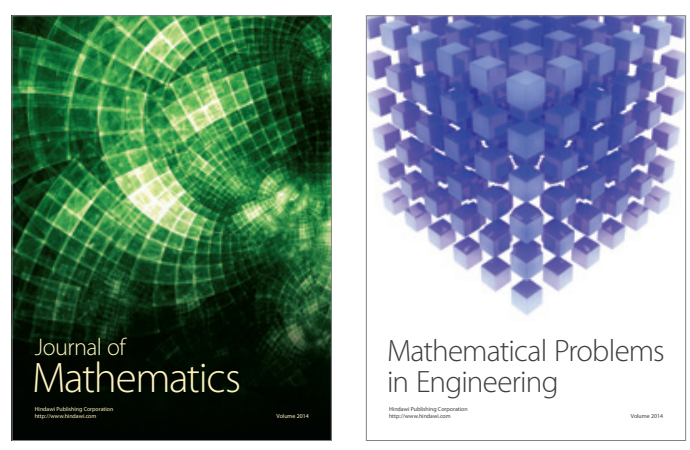

Mathematical Problems in Engineering
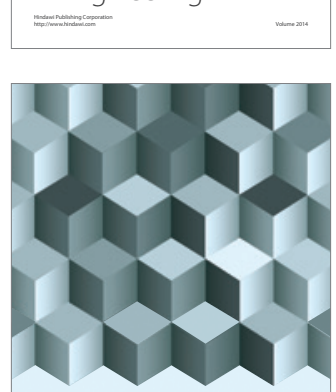

Journal of

Function Spaces
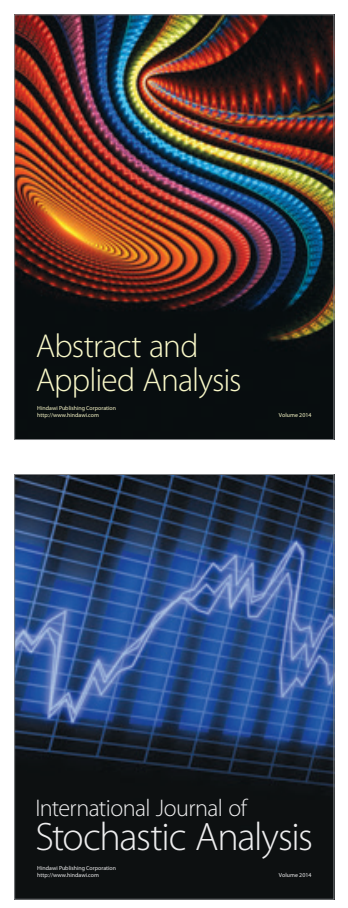

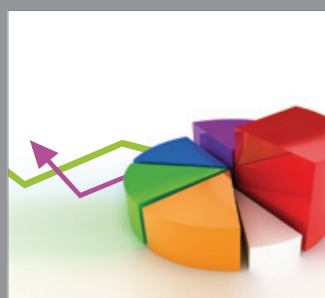

ournal of

Probability and Statistics

Promensencen
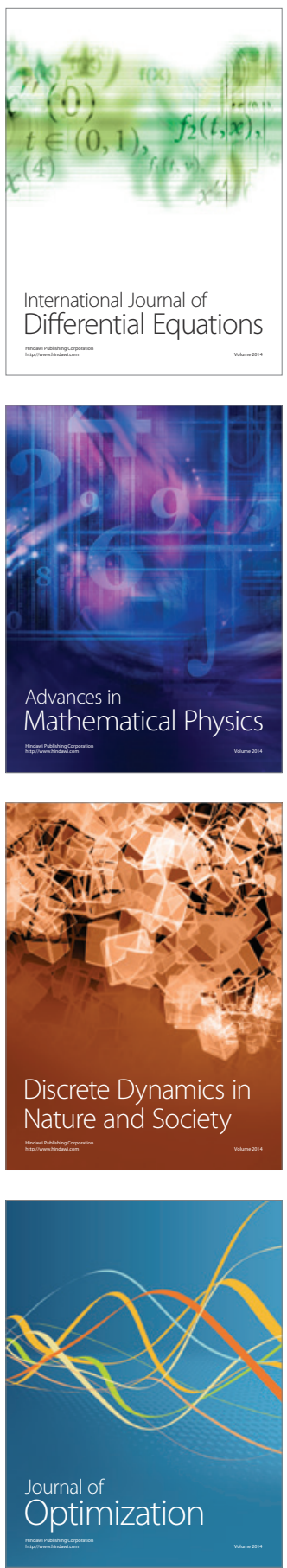